TRANSACTIONS OF THE

AMERICAN MATHEMATICAL SOCIETY

Volume 350, Number 3, March 1998, Pages 1103-1128

S $0002-9947(98) 02085-6$

\title{
GREEN'S FUNCTION, HARMONIC TRANSPLANTATION, AND BEST SOBOLEV CONSTANT IN SPACES OF CONSTANT CURVATURE
}

\author{
C. BANDLE, A. BRILLARD, AND M. FLUCHER
}

\begin{abstract}
We extend the method of harmonic transplantation from Euclidean domains to spaces of constant positive or negative curvature. To this end the structure of the Green's function of the corresponding LaplaceBeltrami operator is investigated. By means of isoperimetric inequalities we derive complementary estimates for its distribution function. We apply the method of harmonic transplantation to the question of whether the best Sobolev constant for the critical exponent is attained, i.e. whether there is an extremal function for the best Sobolev constant in spaces of constant curvature. A fairly complete answer is given, based on a concentration-compactness argument and a Pohozaev identity. The result depends on the curvature.
\end{abstract}

\section{INTRODUCTION}

Harmonic transplantation is a device to construct test functions for variational problems of the form

$$
J[D]:=\inf _{v \in H_{0}^{1}(D)} \frac{\int_{D}|\nabla v(x)|^{2} d x}{\left(\int_{D}|v(x)|^{p} d x\right)^{2 / p}} .
$$

$D$ is a domain in $\mathbb{R}^{N}$ with smooth boundary. Harmonic transplantation replaces conformal transplantation for simply connected planar domains. If $f: B \rightarrow D$ is a Riemann map from the unit disk to $D$ and $u$ an arbitrary function defined in $B$, then the function $U=u \circ f^{-1}$ is called the conformal transplantation of $u$ into $D$. It has the following fundamental properties. The Dirichlet integral is invariant under conformal transplantation, i.e.

$$
\int_{B}|\nabla u|^{2} d x=\int_{D}|\nabla U|^{2} d x
$$

Moreover, if $u$ is positive, radial and $\Phi \geq 0$, then

$$
r^{2}(x) \int_{B} \Phi(u) d x \leq \int_{D} \Phi(U) d x,
$$

where $r(x)$ denotes the harmonic radius of $D$ at $x$ defined below. Conformal transplantation has numerous applications in mathematical physics and function theory (Pólya and Szegö [19], Pólya and Schiffer [18], Hersch [14], and [5]). In particular it has been used to estimate the domain functional $J[D]$ from above, in contrast

Received by the editors June 15, 1996.

1991 Mathematics Subject Classification. Primary 49S05, 35J25, 35J65.

Key words and phrases. Green's function, harmonic transplantation, best Sobolev constant.

(C)1998 American Mathematical Society 
to symmetrization techniques which yield estimates from below. Note that every minimizer in a ball $B_{R}:=\{x:|x|<R\}$ is positive and radial. Harmonic transplantation as proposed by Hersch [14] generalizes conformal transplantation to higher dimensions and to multiply connected domains. Its definition involves the Green's function $G(x, y)$ of $D$ with Dirichlet boundary conditions

$$
\begin{aligned}
\Delta_{x} G(x, y) & =-\delta_{y} \text { in } D, \\
G(x, y) & =0 \text { on } \partial D .
\end{aligned}
$$

The Green's function can be decomposed into a fundamental singularity and a regular part:

$$
\begin{aligned}
G(x, y) & =\gamma(F(|x-y|)-H(x, y)), \\
\gamma & := \begin{cases}\frac{1}{2 \pi} & (N=2), \\
\frac{\Gamma(N / 2)}{2(N-2) \pi^{N / 2}} & (N \geq 3),\end{cases} \\
F(s) & := \begin{cases}-\log (s) & (N=2), \\
s^{2-N} & (N \geq 3) .\end{cases}
\end{aligned}
$$

The regular part $H(x, y)$ is harmonic in both variables. Its boundary values are $F(|x-y|)$. It is regular for every $x \in D$, in particular at $x=y$. The harmonic radius $r(y)$ of $D$ at $y$ is defined by [6]

$$
r(y):= \begin{cases}\exp (-H(y, y)) & (N=2) \\ H^{\frac{1}{2-N}}(y, y) & (N \geq 3)\end{cases}
$$

Let $D$ be a domain in $\mathbb{R}^{N}$ and fix $y \in D$. If $u: B_{r(y)} \rightarrow \mathbb{R}^{+}$is a radial function and $\mu$ is defined by $u(x)=\mu\left(G_{B_{r(y)}}(0, x)\right)$, then

$$
U(x):=\mu\left(G_{D}(x, y)\right)
$$

defines the harmonic transplantation of $u$ into $D$. It has the properties

$$
\begin{aligned}
\int_{B_{r(y)}}|\nabla u|^{2} d x & =\int_{D}|\nabla U|^{2} d x, \\
\int_{D} \Phi(U) d x & \geq \int_{B_{r(y)}} \Phi(u) d x
\end{aligned}
$$

for every positive function $\Phi$. By means of harmonic transplantation Hersch [14] proved that the lowest eigenvalue $\lambda_{1}$ of the Dirichlet Laplacian satisfies

$$
\lambda_{1}(D) \leq \lambda_{1}\left(B_{\max r}\right)
$$

In fact, if we take for $u$ the first eigenfunction in $B_{\max r}$, Rayleigh's principle together with (2) implies

$$
\lambda_{1}(D) \leq \frac{\int_{D}|\nabla U|^{2} d x}{\int_{D}|U|^{2} d x} \leq \frac{\int_{B_{\max r}}|\nabla u|^{2} d x}{\int_{B_{\max r}}|u|^{2} d x}=\lambda_{1}\left(B_{\max r}\right) .
$$


In this paper we extend harmonic transplantation to spaces of constant curvature. We show that the results for the flat case carry over to these spaces. This is possible because the isoperimetric inequality relating volume and surface, which is the key for (2), also holds in these spaces. The inequalities obtained by means of harmonic transplantation are complementary to those obtained by symmetrization. Also Schwarz symmetrization can be extended to spaces of constant curvature (Baernstein [4], Sperner [21], Pólya and Szegö [19]). By scaling we can assume that a space of constant curvature has curvature 0,1 or -1 . Thus we restrict our attention to domains in $\mathbb{R}^{N}, S^{N}$ and $H^{N}$ respectively (Euclidean, spherical and hyperbolic domains).

In Section 2 of our paper we describe the canonical representation of spaces of constant curvature obtained by stereographic projection, and we study their conformal automorphisms. In Section 3 and 4 we construct the Green's function and the harmonic radius of spaces of constant curvature. Section 5 deals with the relation between the Green's function and the capacity of sets. We derive pointwise estimates for the distribution function of the capacity potential and the Green's function. This leads to $L^{p}$-estimates for both functions. In Section 6 we introduce harmonic transplantation for spaces of constant curvature. Finally, in Section 7 we apply it to the nonlinear Dirichlet problem with critical Sobolev exponent in variational form. The problem of estimating the best Sobolev constants of manifolds has been raised by Aubin [3]. Our main interest is to determine whether the best Sobolev constant is attained or not. To this end we need to extend the concentration-compactness alternative of P.L. Lions [15] to conformal metrics. It turns out that the answer depends on the curvature. Nonexistence of minimizers for negatively curved spaces is proved by means of a Pohozaev identity.

\section{Canonical Representation and isometries of $S^{N}$ and $H^{N}$}

Let $S^{N}:=\left\{x \in \mathbb{R}^{N+1}:|x|=1\right\}$. The stereographic projection

$$
\Pi: S^{N} \rightarrow \mathbb{R}^{N}:=\left\{x \in \mathbb{R}^{N+1}: x_{N+1}=0\right\}
$$

maps a point $P^{\prime} \in S^{N}$ into the intersection $P \in \mathbb{R}^{N}$ of the line joining $P^{\prime}$ and the north pole $N=(0,0, \ldots, 1)$ with $\mathbb{R}^{N}$. It is a one-to-one conformal transformation which transforms the line element of $S^{N}$ into

$$
d s_{+}:=p_{+}(x)|d x|, \quad p_{+}(x):=\frac{2}{1+|x|^{2}} .
$$

The pair $\left(D, d s_{+}\right)$with $D \subset \mathbb{R}^{N}$ is called the canonical representation of the domain $D^{\prime}=\Pi^{-1}(D)$ in $S^{N}$. For our purpose it is convenient to work with this representation. For the hyperbolic space $H^{N}$ we choose the canonical representation given by the unit ball $B=\left\{x \in \mathbb{R}^{N}:|x|<1\right\}$ endowed with the metric

$$
d s_{-}:=p_{-}(x)|d x|, \quad p_{-}(x):=\frac{2}{1-|x|^{2}} .
$$

Conformal transformations of the plane are holomorphic mappings, whereas in higher dimensions the only possibilities are rotations, dilations, inversions $x \mapsto$ $x^{*}:=\frac{x}{\left|x^{2}\right|}$ and their compositions. They are commonly called Möbius transformations. Under a conformal transformation

$$
x=g(\xi), \quad \xi=f(x)
$$


the line element transforms according to

$$
d s_{ \pm}=p_{ \pm}(g(\xi))\left|g^{\prime}(\xi)\right| d \xi
$$

where $g^{\prime}(\xi)$ is the matrix $\left(\frac{\partial x_{i}}{\partial \xi_{j}}\right)$ and $\left|g^{\prime}(\xi)\right|=\left|\operatorname{det} g^{\prime}(\xi)\right|^{1 / N}$. Note that $\left|g^{\prime}(\xi)\right|$ is the linear scale at $x$ and that $g^{\prime}(\xi) /\left|g^{\prime}(\xi)\right|$ belongs to the orthogonal group $\mathbf{O}(N)$. Now we restrict our attention to isometries.

2.1. Isometries of $S^{N}$. Every isometry $f$ of $S^{N}$ is a rotation $R_{Y}$ transforming the point $Y \in S^{N}$ into the south pole. Set $y=\Pi(Y)$ and $y^{*}=\frac{y}{|y|^{2}}$. The opposite point $\Pi(-Y)=-y^{*}$ is mapped into the north pole. A Möbius transformation with $f(y)=0$ and $f\left(-y^{*}\right)=\infty$ is of the form

$$
f(x)=\lambda M\left(\left(x+y^{*}\right)^{*}-\left(y+y^{*}\right)^{*}\right)
$$

with $\lambda>0$ and a constant orthogonal matrix $M$ (Ahlfors [1, p. 21]). We want to determine $\lambda$. By (3) we have

$$
p_{+}(x)=p_{+}(f(x))\left|f^{\prime}(x)\right| .
$$

Using the identity

$$
\left|(a+b)^{*}-b^{*}\right|=\frac{|a|}{|a+b||b|}
$$

we get

$$
|f(x)|=\lambda \frac{|x-y|}{\left|x+y^{*}\right|\left|y+y^{*}\right|} .
$$

If a Möbius map satisfies $f(y)=0$, then (Ahlfors [1])

$$
|f(x)|=|f(x)-f(y)|=\left|f^{\prime}(x)\right|^{1 / 2}\left|f^{\prime}(y)\right|^{1 / 2}|x-y| .
$$

As $x \rightarrow y$ we get

$$
\left|f^{\prime}(y)\right|=\frac{\lambda}{\left|y+y^{*}\right|^{2}} .
$$

Evaluation of (4) at $x=y$ yields

$$
\lambda=\frac{\left|y+y^{*}\right|^{2}}{1+|y|^{2}}=\frac{1+|y|^{2}}{|y|^{2}} .
$$

Hence the map $\Pi \circ R_{Y} \circ \Pi^{-1}$ is of the form

$$
f(x)=\frac{1+|y|^{2}}{|y|^{2}} M\left(\left(x+y^{*}\right)^{*}-\left(y+y^{*}\right)^{*}\right)
$$

with $M \in \mathbf{O}(N)$. From (5) and (6) we get

$$
|f(x)|=\frac{|x-y|}{\left|x+y^{*}\right||y|} .
$$

The image of the sphere $\{\xi:|\xi|=c\}$ is

$$
\left\{x: \frac{|x-y|}{\left|x+y^{*}\right|}=c|y|\right\}
$$

i.e. a sphere of Apollonius with limit points $y$ and $-y^{*}$. 
2.2. Isometries of $H^{N}$. Every isometry of $H^{N}$ is a conformal map $f: B \rightarrow B$. The general form of such a map is (Ahlfors [1])

$$
f(x)=T_{y}(x):=M \frac{\left(1-|y|^{2}\right)(x-y)-|x-y|^{2} y}{[x, y]^{2}}
$$

with $M \in \mathbf{O}(N)$ and

$$
[x, y]:=1+|x|^{2}|y|^{2}-2 x \cdot y .
$$

Note that $T_{y}(y)=0$. A laborious computation yields

$$
\left|T_{y}(x)\right|=\frac{|x-y|}{[x, y]} \text { and }\left|T_{y}^{\prime}(x)\right|=\frac{1-|y|^{2}}{[x, y]^{2}}
$$

Also the isometries of $H^{N}$ transform spheres into spheres.

\section{GREEN's FUnCTION FOR SPHERICAL AND HYPERBOLIC DOMAINS}

The gradient, Dirichlet integral, and Laplace-Beltrami operator corresponding to a conformal metric $d s=p(x)|d x|$ are

$$
\begin{aligned}
\nabla_{p} u & :=\frac{\nabla u}{p}, \\
D(u) & :=\int_{D}\left|\nabla_{p} u\right|^{2} p^{N} d x=\int_{D}|\nabla u|^{2} p^{N-2} d x, \\
L u & :=p^{-N} \operatorname{div}\left(p^{N-2} \nabla u\right) .
\end{aligned}
$$

Some computations involving Hölder's and Sobolev's inequality are more transparent when written in terms of $\nabla_{p}$. The Sobolev space $H_{0}^{1}\left(D, p^{N} d x\right)$ is defined by the norm $\|v\|:=\sqrt{D(v)}$. Harmonic functions and Green's functions are readily extended to conformal metrics. The Green's function $G(x, y)=G_{D}(x, y)$ solves

$$
\begin{aligned}
\operatorname{div}_{x}\left(p^{N-2} \nabla_{x} G(x, y)\right) & =-\delta_{y} \text { in } D, \\
G(x, y) & =0 \text { on } \partial D
\end{aligned}
$$

for every $y \in D$. For sufficiently smooth domains a unique Green's function exists in the classical sense (Miranda [16, p. 106]). An important tool for the construction of Green's functions is their conformal invariance (Proposition 2 below). We employ it to determine the singularity of the Green's function. In the rest of this section we consider Euclidean, spherical and hyperbolic domains for which the conformal factor $p$ is either $1, p_{+}$or $p_{-}$. Radial singular solutions of $L F=0$ solve the ordinary differential equation

$$
\left(r^{N-1} p^{N-2}(r) F^{\prime}(r)\right)^{\prime}=0
$$

for $r:=|x|>0$, where $p(r):=p(x)$. Hence

$$
F^{\prime}(r)=\frac{c}{p^{N-2}(r) r^{N-1}}=b_{-N+1} r^{-N+1}+b_{-N+3} r^{-N+3}+\ldots+b_{N-3} r^{N-3} .
$$

We choose the constant $c$ such that

$$
F(r)=\left\{\begin{array}{l}
-\log (r), \\
(2 r)^{-N+2}+a_{-N+4} r^{-N+4}+\ldots+a_{-1} r^{-1}+a_{1} r+\ldots+a_{N-2} r^{N-2}, \\
(2 r)^{-N+2}+a_{-N+4} r^{-N+4}+\ldots+a_{0} \log (r)+\ldots+a_{N-2} r^{N-2}
\end{array}\right.
$$


for $N=2, N=3,5, \ldots$, and $N=4,6, \ldots$ respectively.

Examples. 1. If $N=3$ then $F_{ \pm}(r)=\frac{1}{2 r} \mp \frac{r}{2}$.

2. If $N=4$ then $F_{ \pm}(r)=\frac{1}{4 r^{2}} \mp \log (r)-\frac{r^{2}}{4}$.

3. For $N \geq 3$ we have

$$
F_{-}(r)=(N-2) \int_{r}^{1} p_{-}^{2-N}(s) s^{1-N} d s .
$$

Obviously $F$ decreases and satisfies

$$
\lim _{r \rightarrow 0} F(r)=\infty, \lim _{r \rightarrow \infty} F_{+}(r)=-\infty, \lim _{r \rightarrow 1} F_{-}(r)=0 .
$$

The function $s(x):=\gamma F(|x|)$ with $\gamma$ defined in (1) is a fundamental solution of

$$
L s=-\delta_{0} .
$$

If the singularity is not at the origin we have:

Lemma 1. The functions $s(x, y):=\gamma F(x, y)$ with

$$
F(x, y):= \begin{cases}F_{+}\left(\frac{|x-y|}{\left|x+y^{*}\right||y|}\right) & \left(p=p_{+}\right), \\ F_{-}\left(\frac{|x-y|}{[x, y]}\right) & \left(p=p_{-}\right)\end{cases}
$$

are fundamental solutions with singularities in $x=y$.

Proof. Let $F(|\xi|)$ be a radial fundamental solution. If $f(x)$ is an isometry with $f(y)=0$, then $w(x):=F(|f(x)|)$ solves $L_{x} w=-\delta_{y}$. The assertion now follows from (7) and (8).

The function $F(x, y)$ is symmetric in $x$ and $y$. As for Euclidean domains, the Green's function can be decomposed into

$$
G(x, y)=\gamma(F(x, y)-H(x, y))
$$

where

$$
\begin{aligned}
L_{x} H(x, y) & =0 \text { in } D, \\
H(x, y) & =F(x, y) \text { for } x \in \partial D .
\end{aligned}
$$

On a ball $B_{R}$ the Green's function with singularity at the origin is

$$
G(x, 0)=\gamma(F(|x|)-F(R)) .
$$

The Green's function of spherical and hyperbolic domains shares most properties of the Euclidean Green's function.

Proposition 2. 1. Positivity: $G(x, y)$ is positive in $D$.

2. Symmetry: $G(x, y)=G(y, x)$ for every $x$ and $y$ in $D$.

3. Monotonicity: If $D_{1} \subset D_{2}$, then $G_{D_{1}}(x, y) \leq G_{D_{2}}(x, y)$ in $D_{1}$.

4. Isometric invariance: If $f: D_{1} \rightarrow D_{2}$ is a conformal isometry, then

$$
G_{D_{2}}(f(x), f(y))=G_{D_{1}}(x, y) .
$$


5. Superposition principle: The solution of the problem

$$
\begin{aligned}
L u & =-f \text { in } D, \\
u & =0 \text { on } \partial D
\end{aligned}
$$

is

$$
u(x)=\int_{D} G(x, y) f(y) p^{N}(y) d y .
$$

The proof is as in the Euclidean case.

\section{HARMONIC RADIUS OF SPHERICAL AND HYPERBOLIC DOMAINS}

Definition 3 (Harmonic radius). The harmonic radius of $(D, d s)$ at $y$ is the solution of

$$
F(r(y))=H(y, y)
$$

with $F$ and $H$ as in Section 3 .

Since $F$ is monotone, $r(y)$ is well defined. Moreover it is independent of the particular solution $F$ of (9). The above definition is equivalent to

$$
F(r(y))=\lim _{x \rightarrow y}\left(F(x, y)-\gamma^{-1} G(x, y)\right) .
$$

For the unit ball $B \subset S^{N}$ we have $r_{+}(0)=1$. In $H^{N}$ we have $r_{-} \equiv 1$. Continuity of the regular part of the Green's function implies continuity of the harmonic radius. On the boundary $H(x, y)=F(x, y)(x \in \partial D)$, and consequently

$$
\begin{aligned}
\lim _{y \rightarrow x \in \partial D} H(x, y) & =\infty, \\
r(y) & =0 \text { on } \partial D .
\end{aligned}
$$

By definition $r(y)$ is positive in $D$ and assumes its maximum provided $D$ is bounded. The points in $D$ where $r(y)$ achieves its maximum are called harmonic centers. As in [6] the properties of the Green's function easily translate into the following ones for the harmonic radius.

Proposition 4. 1. Positivity: $r(y)$ is positive in D.

2. Monotonicity: If $D_{1} \subset D_{2}$ then $r_{1}(y) \leq r_{2}(y)$ in $D_{1}$.

3. The spherical (hyperbolic) radius $r_{ \pm}$and the Euclidean harmonic radius $r_{0}$ of a two-dimensional domain are related by

$$
r_{ \pm}(y)=\frac{1}{2} p_{ \pm}(y) r_{0}(y) \text {. }
$$

Proof. We only need to prove the last claim. The Green's function of a spherical domain is of the form

$$
G_{+}(x, y)=\frac{1}{2 \pi}\left(-\log \frac{|x-y|}{|y|\left|x+y^{*}\right|}-H_{+}(x, y)\right)
$$

while the corresponding Euclidean Green's function is

$$
G_{0}(x, y)=\frac{1}{2 \pi}\left(-\log |x-y|-H_{0}(x, y)\right) .
$$

In view of the Dirichlet boundary condition for the Green's function and the fact that in two dimensions harmonicity is independent of $p$, we have

$$
\frac{H_{+}(x, y)}{4}-\log \left(|y|\left|x+y^{*}\right|\right)=H_{0}(x, y)
$$


for $x, y \in D$. If $x=y$ we obtain

$$
-\log r_{+}(y)-\log \left(1+|y|^{2}\right)=\log r_{0}(y),
$$

which completes the proof. The argument for hyperbolic domains is similar.

The two-dimensional case is special in several respects. If $D$ is simply connected, then the function $U(y):=-\log \left(r_{0}(y)\right)$ is a solution of Liouville's equation [6]:

$$
\Delta U=4 e^{2 U} \text { in } D \text {. }
$$

Therefore $V(y):=-\log (r(y))=U(y)-\log (p(y) / 2)$ solves

$$
\Delta V=\Delta U-\Delta \log p=\Delta U+K p^{2},
$$

where $K$ denotes the Gaussian curvature of $(D, p|d x|)$. Thus

$$
\begin{aligned}
\Delta V & =p^{2}\left(e^{2 V}+K\right) \text { or } \\
L V & =e^{2 V}+K \text { in } D .
\end{aligned}
$$

Since $V(y) \rightarrow \infty$ as $y \rightarrow \partial D$, it is the maximal solution of (11). The harmonic radii for simply connected domains in $S^{2}$ and $H^{2}$ have been defined in [5] together with extensions for domains on general two-dimensional surfaces.

\section{CAPACITY}

The capacity of a set $A \subset D$ with respect to $D$ is defined as

$$
\operatorname{cap}_{D}(A):=\inf \left\{\int_{D}|\nabla v|^{2} p^{N-2} d x: v \in H_{0}^{1}\left(D, p^{N} d x\right), v \geq 1 \text { on } A\right\} .
$$

The corresponding Euler equation is

$$
\begin{aligned}
L k & =0 \text { in } D \backslash A, \\
k & =1 \text { on } \partial A, \\
k & =0 \text { on } \partial D .
\end{aligned}
$$

Its solution is called the capacity potential of $A$. In the radial case the capacity is

$$
\operatorname{cap}_{B_{R}}\left(B_{r}\right)=\frac{1}{\gamma(F(r)-F(R))} .
$$

An immediate consequence of the variational characterization of the capacity is the following monotonicity property. If $A \subset A_{1}$ and $D_{1} \subset D$, then

$$
\operatorname{cap}_{D_{1}}\left(A_{1}\right) \geq \operatorname{cap}_{D}(A) .
$$

The capacity is a conformal invariant. For every function $f: D \rightarrow \mathbb{R}$ let

$$
D_{f}(t):=\{x \in D: f(x)>t\} .
$$

A simple calculation shows that

$$
\operatorname{cap}_{D}\left(D_{k}(t)\right)=\frac{1}{t} \int_{\{k=t\}}|\nabla k| p^{N-2} d \mathcal{H}^{N-1}=\frac{\operatorname{cap}_{D}(A)}{t}
$$

where $d \mathcal{H}^{N-1}$ denotes the $(N-1)$-dimensional Hausdorff measure. For the Green's function one has

$$
\operatorname{cap}_{D}\left(D_{G(\cdot, y)}(t)\right)=\frac{1}{t} \int_{\{G(\cdot, y)=t\}}|\nabla G(\cdot, y)| p^{N-2} d \mathcal{H}^{N-1}=\frac{1}{t} .
$$


From this identity we get the following relation between the harmonic radius and the capacity:

$$
F(r(0))=\lim _{\rho \rightarrow 0} \gamma^{-1}\left(s(\rho)-\operatorname{cap}_{D}\left(B_{\rho}\right)\right) .
$$

5.1. Inequalities for the distribution function of the capacity potential. Bernstein [7] extended the classical isoperimetric inequality to domains on $S^{2}$. The generalization to other spaces of constant curvature is due to Schmidt [20]. For every domain $(D, p|d x|)$ denote by

$$
\begin{aligned}
m(D) & :=\int_{D} p^{N} d x \\
o(\partial D) & :=\int_{\partial D} p^{N-1} d \mathcal{H}^{N-1}
\end{aligned}
$$

its volume and surface with respect to the metric $p|d x|$. For every measurable set $A$ in a space of constant curvature, denote by $A^{*}:=B_{R}$ the geodesic ball of the same volume. Schmidt's isoperimetric inequality states that

$$
o(\partial A) \geq o\left(\partial A^{*}\right) .
$$

It is a key tool for the sequel. Let $m(t):=m\left(D_{k}(t)\right)$ be the distribution function of the capacity potential $k(x)$ defined in (12). It is decreasing and satisfies $m(1)=$ $m(A), m(0)=m(D)$. By the coarea formula

$$
-\frac{d m(t)}{d t}=\int_{\partial D_{k}(t)}|\nabla k|^{-1} p^{N} d \mathcal{H}^{N-1} .
$$

In view of (15) and Schwarz's inequality

$$
\begin{aligned}
-\frac{d m(t)}{d t} & =\int_{\partial D_{k}(t)} p^{N-1} \frac{p}{|\nabla k|} d \mathcal{H}^{N-1} \int_{\partial D_{k}(t)} p^{N-1} \frac{|\nabla k|}{p} d \mathcal{H}^{N-1} \operatorname{cap}_{D}^{-1}(A) \\
& \geq\left(\int_{\partial D_{k}(t)} p^{N-1} d \mathcal{H}^{N-1}\right)^{2} \operatorname{cap}_{D}^{-1}(A) \\
& =o^{2}\left(\partial D_{k}(t)\right) \operatorname{cap}_{D}^{-1}(A) .
\end{aligned}
$$

Together with Schmidt's isoperimetric inequality (17) we get

$$
-\frac{d m(t)}{d t} \geq o^{2}\left(\partial D_{k}^{*}(t)\right) \operatorname{cap}_{D}^{-1}(A) .
$$

The radius $R(t)$ of $D_{k}^{*}(t)$ is determined by

$$
m(t)=|\partial B| \int_{0}^{R(t)} s^{N-1} p^{N}(s) d s .
$$

Differentiation yields

$$
-\frac{d m(t)}{d t}=-|\partial B| R^{N-1}(t) p^{N}(R(t)) \frac{d R(t)}{d t} .
$$

In terms of $R(t)$ the surface of the symmetrized level sets of the capacity potential is

$$
o\left(\partial D_{k}^{*}(t)\right)=|\partial B| p^{N-1}(R(t)) R^{N-1}(t) .
$$

Inserting this expression into (18), we find that

$$
-\frac{d R(t)}{d t} \geq|\partial B| R^{N-1}(t) \operatorname{cap}_{D}^{-1}(A) p^{N-2}(R(t)) .
$$


Equality holds if $D$ and $A$ are concentric balls. This inequality implies the isoperimetric inequality for the capacity in spaces of constant curvature (Pólya and Szegö [19]).

Lemma 5 (Isoperimetric inequality for capacity). Let $A \subset D$ in a space of constant curvature. Then

$$
\operatorname{Cap}_{D}(A) \geq \operatorname{Cap}_{D^{*}}\left(A^{*}\right)
$$

Proof. From (20) it follows that

$$
\frac{1}{\operatorname{cap}_{D}(A)} \leq \frac{1}{|\partial B|} \int_{R(1)}^{R(0)} \frac{d R}{R^{N-1} p^{N-2}(R)}=\frac{1}{\operatorname{cap}_{D^{*}}\left(A^{*}\right)}
$$

by definition of $R(t)$.

We denote by $A_{1}^{*}=B_{R_{1}}$ the ball for which

$$
\operatorname{cap}_{D^{*}}\left(A_{1}^{*}\right)=\operatorname{cap}_{D}(A) .
$$

By Lemma 5, monotonicity of the capacity (14), and (13) we have

$$
R_{1} \geq R(1) \text {. }
$$

Let $k_{1}(x)$ be the corresponding capacity potential in $D^{*} \backslash A_{1}^{*}$. Similarly we introduce the ball $D_{0}^{*}=B_{R_{0}}$ for which

$$
\operatorname{cap}_{D_{0}^{*}}\left(A^{*}\right)=\operatorname{cap}_{D}(A) .
$$

By the same reasoning as above we have

$$
R_{0} \leq R(0)
$$

Lemma 6. Let $k_{0}(x)$ be the capacity potential of $A^{*}$ with respect to $D_{0}^{*}$. Then the distribution functions $m_{1}(t)$ and $m_{0}(t)$ of $k_{1}(x)$ and $k_{0}(x)$ satisfy

$$
m_{0}(t) \leq m(t) \leq m_{1}(t) .
$$

Proof. From (20) we get for $t_{0} \leq t_{1}$ :

$$
\int_{R\left(t_{1}\right)}^{R\left(t_{0}\right)} \frac{d R}{R^{N-1} p^{N-2}(R)} \geq \frac{|\partial B|\left(t_{1}-t_{0}\right)}{\operatorname{cap}_{D}(A)} .
$$

Denote by $R_{0}(t)$ and $R_{1}(t)$ the radii of the balls of volumes $m_{0}(t)$ and $m_{1}(t)$. By definition $\operatorname{cap}_{D_{0}^{*}}\left(A^{*}\right)=\operatorname{cap}_{D^{*}}\left(A_{1}^{*}\right)=\operatorname{cap}_{D}(A)$. Thus $(21)$ is an equality if $R(t)=R_{0}(t)$ or $R(t)=R_{1}(t)$. The assertion follows from $R_{1}(0)=R(0)$ and $R_{0}(1)=R(1)$.

Lemma 7 ( $L^{q}$-estimates for the capacity potential). For every $q>0$ one has

$$
\int_{D_{0}^{*} \backslash A^{*}} k_{0}^{q} d x \leq \int_{D \backslash A} k^{q} d x \leq \int_{D_{0}^{*} \backslash A_{1}^{*}} k_{1}^{q} d x+m\left(A_{1}^{*}\right)-m(A) .
$$

Proof. We have

$$
\int_{D \backslash A} k^{q} d x=-\int_{0}^{1} t^{q} \frac{d m(t)}{d t} d t=-m(1)+q \int_{0}^{1} t^{q-1} m(t) d t .
$$

The claim follows from Lemma 6 and $m_{0}(1)=m(1)$. 
5.2. Inequalities for the distribution function of the Green's function. Similar results hold for the distribution function $m(t)$ of the Green's function $G(x, y)$. By definition of the Green's function we have

$$
-\frac{d m}{d t}(t)=\int_{\partial D(t)} \frac{p}{|\nabla G|} p^{N-1} d \mathcal{H}^{N-1}=1 .
$$

As in the previous section, this leads to

$$
-\frac{d R(t)}{d t} \geq|\partial B| R^{N-1}(t) p^{N-2}(R(t))
$$

with $R(t)$ given by (19). Integration yields

$$
\gamma\left(F\left(R\left(t_{1}\right)\right)-F\left(R\left(t_{0}\right)\right)\right) \geq t_{1}-t_{0}
$$

for every $t_{0} \leq t_{1}$. Equality holds in a ball with the singularity at the center. From this inequality we deduce the analogue of Lemma 6 for the Green's function.

Lemma 8. Let $m(t), m_{1}(t)$, and $m_{0}(t)$ be the distribution functions of $G_{D}(x, y)$, $G_{D^{*}}(x, 0)$, and $G_{B_{r(y)}}(x, 0)$ respectively. Then

$$
m_{0}(t) \leq m(t) \leq m_{1}(t) .
$$

Proof. From (22) we have

$$
\int_{R\left(t_{1}\right)}^{R\left(t_{0}\right)} \frac{d R}{R^{N-1} p^{N-2}(R)} \geq|\partial B|\left(t_{1}-t_{0}\right)
$$

with equality for the radially symmetric Green's function. Setting $t_{0}=0$ and observing that $R(0)$ is the radius of $D^{*}$, we get $R\left(t_{1}\right) \leq R_{1}\left(t_{1}\right)$ for all $t_{1}$, whence $m(t) \leq m_{1}(t)$. For the second estimate we let $R\left(t_{1}\right) \rightarrow 0$ and use the fact that

$$
t_{1}=\gamma\left(F\left(R\left(t_{1}\right)\right)-H(y, y)\right)=o(1) \quad \text { as } \quad t_{1} \rightarrow \infty .
$$

Lemma 9 ( $L^{q}$-estimates for the Green's function). If $N=2$ and $q>0$ or $N \geq 3$ and $0<q<N /(N-2)$, then

$$
\int_{B_{\max r}} G_{B_{\max r}}^{q}(x, 0) p^{N} d x \leq \int_{D} G_{D}^{q}(x, y) p^{N} d x \leq \int_{D^{*}} G_{D^{*}}^{q}(x, 0) p^{N} d x .
$$

Proof. The proof is essentially the same as for the capacity potential.

The upper bound for the Green's function in $\mathbb{R}^{N}$ goes back to Weinberger [23]. The corresponding lower bound can be found in [5]. As an application we derive a lower bound for the torsion function. Since

$$
u(x)=\int_{D} G(x, y) p^{N}(y) d y
$$

solves the problem

$$
\begin{aligned}
L u & =-1 \text { in } D, \\
u & =0 \text { on } \partial D,
\end{aligned}
$$

Lemma 9 with $q=1$ implies

$$
\int_{0}^{\max r} \frac{d s}{s^{N-1} p^{N-2}} \int_{0}^{s} p^{N} t^{N-1} d t \leq \max _{x \in D} u(x) \leq \int_{0}^{R^{*}} \frac{d s}{s^{N-1} p^{N-2}} \int_{0}^{s} p^{N} t^{N-1} d t
$$


where

$$
m\left(B_{R^{*}}\right)=m(D)
$$

For $\mathbb{R}^{2}$ this result goes back to Pólya and Szegö [19]; for $\mathbb{R}^{N}$ to Payne [17].

Lemma 10 (Isoperimetric property of harmonic radius). Among all domains of given volume the maximum of the harmonic radius is maximal for balls.

Proof. Assume that $r(y)$ assumes its maximum at the origin. By Lemma 5 and the definition of the harmonic radius we have for $|y| \rightarrow 0$ :

$$
\begin{aligned}
t & =\gamma(F(|y|)-F(r(0))+o(1)) \\
& =\operatorname{cap}_{D}^{-1}\left(D_{G(y, 0)}(t)\right) \\
& \leq \operatorname{cap}_{D^{*}}^{-1}\left(D_{G(y, 0)}^{*}(t)\right) \\
& =\gamma\left(F(|y|)-F\left(R^{*}\right)\right)
\end{aligned}
$$

where $R^{*}$ is the radius of $D^{*}$. This completes the proof, because $R^{*}$ is the maximum of the harmonic radius of $D^{*}$.

\section{HARMONIC TRANSPLANTATION}

The method of harmonic transplantation is based on the following two observations. Let $\nu(t)$ be any differentiable function defined on $\mathbb{R}^{+}$and $G(x, y)$ be the Green's function of $(D, p|d x|)$. Consider

$$
V(x):=\nu(G(x, y)) \text {. }
$$

Since $\nabla V=\nu^{\prime} \nabla G$, it follows from the definition of the Green's function that the Dirichlet integral of $V$ is

$$
D(V)=\int_{0}^{\infty} \nu^{\prime 2}(t) d t \int_{\partial D(t)}|\nabla G| p^{N-2} d \mathcal{H}^{N-1}=\int_{0}^{\infty} \nu^{\prime 2}(t) d t
$$

Thus the Dirichlet integral depends on $\nu(t)$ but not on the domain. In the multiply connected domain $D \backslash A$ we can also consider the function

$$
V(x):=\nu(k(x)),
$$

where $k(x)$ is the capacity potential defined by (12). In this case the Dirichlet integral is

$$
D(V)=\operatorname{cap}_{D}(A) \int_{0}^{\infty} \nu^{\prime 2}(t) d t
$$

The second key is an estimate for integrals of the form

$$
\int_{D} \Phi(V) p^{N} d x
$$

Lemma 11. Let $D$ be a Euclidean or hyperbolic domain or a spherical domain whose volume is smaller than that of a half sphere, let $A \subset D, k(x)$ is the capacity potential of $A$ with respect to $D, k_{0}(x)$ is that of $A^{*}$ with respect to $D_{0}^{*}$ with $D_{0}^{*}$ as in Lemma 6, and $m_{0}(t)$ stands for the distribution function of $k_{0}(x)$. Then

$$
\int_{D \backslash A} \Phi(k(x)) p^{N} d x \geq \int_{D_{0}^{*} \backslash A^{*}} \Phi\left(k_{0}(x)\right) p^{N} d x
$$

for every $\Phi: \mathbb{R}^{+} \rightarrow \mathbb{R}^{+}$. If $\Phi$ is increasing, the inequality holds for arbitrary spherical domains. 
Proof. Integration over level surfaces yields

$$
\int_{D \backslash A} \Phi(k(x)) p^{N} d x=\int_{0}^{1} \Phi(t)\left(-\frac{d m(t)}{d t}\right) d t .
$$

From (19) and (20) we have

$$
-\frac{d m(t)}{d t} \geq\left(|\partial B| p^{N-1}(R(t)) R^{N-1}(t)\right)^{2} \operatorname{cap}_{D}^{-1}(A)
$$

The conclusion follows from $R(t) \geq R_{0}(t)$ (Lemma 6$)$, $\operatorname{cap}_{D}(A)=\operatorname{cap}_{D_{0}^{*}}\left(A^{*}\right)$, and monotonicity of the above right hand side as a function of $R(t)$ for $R(t) \leq 1$ (If $D \subset S^{N}$ we need $m(D) \leq m\left(S^{N}\right) / 2$ to make sure that $\left.R(t) \leq 1\right)$. We integrate by parts to obtain

$$
\int_{0}^{1} \Phi(t)\left(-\frac{d m}{d t}\right) d t=\Phi(0) m(0)-\Phi(1) m(1)+\int_{0}^{1} \Phi^{\prime}(t) m(t) d t .
$$

Since $m(1)=m_{0}(1)$ and $m(t) \geq m_{0}(t)$ (Lemma 6), the assertion is now obvious.

A similar result holds with the capacity potential replaced by the Green's function. A particular case has been treated in Lemma 9. More generally, we have

Lemma 12. Let $D$ be a Euclidean or hyperbolic domain or a spherical domain whose volume is smaller than that of a half sphere. Then

$$
\int_{D} \Phi\left(G_{D}(x, y)\right) p^{N} d x \geq \int_{B_{r(y)}} \Phi\left(G_{B_{r(y)}}(x, y)\right) p^{N} d x
$$

for every $\Phi: \mathbb{R}^{+} \rightarrow \mathbb{R}^{+}$for which $\Phi(G(\cdot, y))$ is integrable. If $\Phi$ is increasing, the inequality holds for arbitrary spherical domains.

Proof. The proof is the same as for Lemma 11, except that inequality (25) has to be replaced by

$$
-\frac{d m(t)}{d t} \geq\left(|\partial B| p^{N-1}(R(t)) R^{N-1}(t)\right)^{2} .
$$

Definition 13 (Harmonic transplantation for spaces of constant curvature). Let $D$ be a space of constant curvature and fix $y \in D$.

1. Consider a geodesic ball $B_{\rho}$ in $\mathbb{R}^{N}, S^{N}$ or $H^{N}$ (not necessarily in the same space as $D$ ) and a radially symmetric function $u: B_{\rho} \rightarrow \mathbb{R}^{+}$. It can be written as $u(x)=\mu\left(G_{B_{\rho}}(x, 0)\right)$. The harmonic transplantation $U$ of $u$ into $D$ is defined by

$$
U(x):=\mu\left(G_{D}(x, y)\right)
$$

2. Consider a radially symmetric function $u: B_{R} \backslash B_{\rho} \rightarrow \mathbb{R}^{+}$defined on an annulus in $\mathbb{R}^{N}, S^{N}$ or $H^{N}$. It can be written as $u(x)=\mu(k(x))$ of the capacity potential of $B_{\rho}$ with respect to $B_{R}$. Let $D \backslash A$ be an arbitrary annulus and $k_{D \backslash A}$ the corresponding capacity potential. Then we define

$$
U(x):=\mu\left(k_{D \backslash A}(x)\right) .
$$

We summarize the relations between $u$ and $U$.

Theorem 14. Let $D$ be a space of constant curvature. Fix $y \in D$. Let $u$ be as in the first part of the above definition with $\rho$ replaced by the harmonic radius $r(y)$ of D. Then 
1. The transplanted function $U$ defined in (27) satisfies

$$
\int_{D}|\nabla U(x)|^{2} p^{N-2} d x=\int_{B_{r(y)}}|\nabla u(x)|^{2} p^{N-2} d x .
$$

2. If $D \subset S^{N}$ we assume $m(D) \leq m\left(S^{N}\right) / 2$ or that $\Phi \circ \mu$ is increasing. Then

$$
\int_{D} \Phi(U(x)) p^{N} d x \geq \int_{B_{r(y)}} \Phi(u(x)) p^{N} d x
$$

for every $\Phi: \mathbb{R}^{+} \rightarrow \mathbb{R}^{+}$.

Proof. The assertions follow from (24) and Lemma 12.

Similarly we infer from Lemma 11:

Theorem 15. Let $D$ be a space of constant curvature and $D \backslash A$ an annulus in $D$. Define $R(1)$ by $m(A)=m\left(B_{R(1)}\right)$ and $R_{0}$ by $\operatorname{Cap}_{B_{R_{0}}}\left(A^{*}\right)=\operatorname{Cap}_{D}(A)$. Let $u: B_{R(1)} \backslash B_{R_{0}} \rightarrow \mathbb{R}$ be a radial function with transplanted function $U$ on $D \backslash A$.

1. The transplanted function $U$ defined in (28) satisfies

$$
\int_{D \backslash A}|\nabla U(x)|^{2} d x=\int_{B_{R(1)} \backslash B_{R_{0}}}|\nabla u(x)|^{2} d x
$$

2. If $D \subset S^{N}$ we assume $m(D) \leq m\left(S^{N}\right) / 2$ or that $\Phi \circ \mu$ is increasing. Then

$$
\int_{D \backslash A} \Phi(U(x)) d x \geq \int_{B_{R(1)} \backslash B_{R_{0}}} \Phi(u(x)) d x .
$$

for every $\Phi: \mathbb{R}^{+} \rightarrow \mathbb{R}^{+}$.

Harmonic transplantation leads to upper bounds for functionals of the form

$$
J[D]:=\inf \left\{\int_{D}|\nabla v(x)|^{2} p^{N-2} d x: v \in H_{0}^{1}\left(D, p^{N} d x\right), \int_{D} \Phi(v) p^{N} d x=1\right\} .
$$

If $\Phi(t)=t^{2}$ then $J[D \backslash A]$ is the first Dirichlet eigenvalue $\lambda_{1}$ of the Laplace-Beltrami operator in the multiply connected domain $D \backslash A$. Theorem 15 implies

$$
\lambda_{1}(D \backslash A) \leq \lambda_{1}\left(B_{R(1)} \backslash B_{R_{0}}\right) .
$$

This extends the Gasser-Hersch inequality [12] to spaces of constant curvature. A further example with $\Phi(t)=t^{\frac{2 N}{N-2}}$ is discussed in the next section.

\section{Best Sobolev Constant for Spherical and hyperbolic domains}

In this section the conformality factor may be an arbitrary positive function $p \in C(\bar{D})$. We study the variational problem

$$
S_{p}(D):=\inf \left\{\frac{\int_{D}|\nabla u|^{2} p^{N-2} d x}{\left(\int_{D}|u|^{2^{*}} p^{N} d x\right)^{2 / 2^{*}}}: u \in H_{0}^{1}\left(D, p^{N} d x\right) \backslash\{0\}\right\}
$$

with $N \geq 3$ and the critical Sobolev exponent

$$
2^{*}:=\frac{2 N}{N-2} .
$$


The infimum $S_{p}(D)$ is called the best Sobolev constant. Alternatively we can minimize the Dirichlet integral among normalized functions

$$
\begin{aligned}
S_{p}(D) & =\inf _{u \in E} \int_{D}|\nabla u|^{2} p^{N-2} d x, \\
E & :=\left\{u \in H_{0}^{1}\left(D, p^{N} d x\right): \int_{D}|u|^{2^{*}} p^{N} d x=1\right\} .
\end{aligned}
$$

If the minimum in (30) is achieved, the minimizer is a positive solution of the Euler equation

$$
\begin{aligned}
L u+S_{p}(D) u^{2^{*}-1} & =0 \text { in } D, \\
u & =0 \text { on } \partial D .
\end{aligned}
$$

We compute the best Sobolev constant for spherical and hyperbolic domains, and we want to know whether the best Sobolev constant is attained, i.e. whether (31) has a nontrivial solution. The best Sobolev constant of all Euclidean domains of dimension $N$ is

$$
S=N(N-2) \pi\left(\frac{\Gamma(N / 2)}{\Gamma(N)}\right)^{2 / N} .
$$

For constant $p$ the ratio in $(29)$ is independent of $p$, hence $S_{p}(D)=S$. More generally we have:

Proposition 16. If $p \in C(\bar{D})$ and $p>0$ in $\bar{D}$, then

$$
S_{p}(D) \leq S .
$$

Proof. Let $x_{0}$ be an arbitrary point in $D$. There are functions $u_{\varepsilon} \in E$ whose Dirichlet integral is arbitrarily close to $S$. For instance

$$
u_{\varepsilon}(x)=c_{\varepsilon} \phi(x)\left(\frac{1}{\varepsilon^{2}+\left|x-x_{0}\right|^{2}}\right)^{\frac{N-2}{2}}
$$

with a cut-off function $\phi$ which is supported in a small neighbourhood of $x_{0}$ and constant near $x_{0}$, has this property. By continuity of $p$ we can replace it by the positive constant $p\left(x_{0}\right)$. The claim follows from the above remark on the case of a constant conformal factor.

7.1. Concentration-compactness alternative and compactness criterion for conformal metrics. By $\mathcal{M}(\bar{D})$ we denote the class of non-negative Borel measures on $\bar{D}$. We say that the sequence $\left(\mu_{\varepsilon}\right)$ tends to $\mu$ in the sense of measures, denoted as $\mu_{\varepsilon} \rightarrow \mu$ in $\mathcal{M}(\bar{D})$, if $\int_{\bar{D}} \phi d \mu_{\varepsilon} \rightarrow \int_{\bar{D}} \phi d \mu$ for every test-function $\phi \in$ $C(\bar{D})$. A point $x$ is called an atom of $\mu$ if $\lim _{\rho \rightarrow 0} \mu\left(B_{x}^{\rho}\right)>0$. The compactness criterion (Theorem 18) is based on the concentration-compactness alternative stated below. It extends the result of P. L. Lions [15] to conformal metrics. For the convenience of the reader a complete proof is included.

Theorem 17. Let $\left(u_{\varepsilon}\right)$ be a sequence in $E$ with

$$
\begin{aligned}
u_{\varepsilon} & \rightarrow u_{0} \text { weakly in } H_{0}^{1}\left(D, p^{N} d x\right), \\
\left|\nabla u_{\varepsilon}\right|^{2} p^{N-2} d x & \rightarrow \mu \text { in } \mathcal{M}(\bar{D}) \\
\left|u_{\varepsilon}\right|^{2^{*}} p^{N} d x & \rightarrow \nu \text { in } \mathcal{M}(\bar{D})
\end{aligned}
$$


Then the limit measures are of the form

$$
\begin{aligned}
& \mu=\left|\nabla u_{0}\right|^{2} p^{N-2} d x+\sum_{j=1}^{J} \mu_{j} \delta_{x_{j}}+\widetilde{\mu}, \mu(\bar{D}) \geq S_{p}(D), \\
& \nu=\left|u_{0}\right|^{2^{*}} p^{N} d x+\sum_{j=1}^{J} \nu_{j} \delta_{x_{j}}, \nu(\bar{D})=1,
\end{aligned}
$$

with $J \in \mathbb{N} \cup\{\infty\}$ and a nonatomic positive measure $\widetilde{\mu}$. The atoms satisfy the Sobolev inequality

$$
\frac{\mu_{j}}{\nu_{j}^{2 / 2^{*}}} \geq S
$$

If $\left(u_{\varepsilon}\right)$ is a minimizing sequence for $S_{p}(D)$, then one of the following statements holds true.

1. Concentration: $\nu=\delta_{x_{0}}$ and $\mu=S \delta_{x_{0}}$ are concentrated at a single point and $u_{0}=0$.

2. Compactness: $u_{\varepsilon} \rightarrow u_{0}$ in $H_{0}^{1}\left(D, p^{N} d x\right), \mu=\left|\nabla u_{0}\right|^{2} p^{N-2} d x$, and $\nu=$ $\left|u_{0}\right|^{2^{*}} p^{N} d x$.

Proof. For a subsequence $u_{\varepsilon} \rightarrow u_{0}$ almost everywhere. By the Brézis-Lieb lemma [8]

$$
\liminf _{\varepsilon \rightarrow 0} \int_{D^{\prime}}\left|u_{\varepsilon}\right|^{2^{*}} p^{N} d x-\int_{D^{\prime}}\left|u_{0}\right|^{2^{*}} p^{N} d x=\liminf _{\varepsilon \rightarrow 0} \int_{D^{\prime}}\left|u_{\varepsilon}-u_{0}\right|^{2^{*}} p^{N} d x
$$

for every subdomain $D^{\prime} \subset D$. Thus

$$
\nu-\left|u_{0}\right|^{2^{*}} p^{N} d x \geq 0 .
$$

Let $\left\{x_{j}: 1 \leq j \leq J\right\}$ be the atoms of this measure, i.e.

$$
\nu=\left|u_{0}\right|^{2^{*}} p^{N} d x+\sum_{j=1}^{J} \nu_{j} \delta_{x_{j}}+\widetilde{\nu}
$$

with nonatomic $\widetilde{\nu} \geq 0$. We will see that actually $\widetilde{\nu}=0$. The set of atoms is countable because $\nu(\bar{D})<\infty$. The proof of (32) is by localization in a neighbourhood of $x_{j}$. As in [11] we use the $n$-harmonic capacity potential as a cut-off function to localize both measures near $x_{j}$. Assume $x_{j}=0$ and define

$$
\phi(x):=\frac{\log (|x| / R)}{\log (\rho / R)} \quad(\rho \leq|x| \leq R),
$$

extended by 1 in $B_{\rho}$ and by 0 outside $B_{R}$. Its conformal energy is

$$
\int_{B_{R}}|\nabla \phi|^{n}=\frac{\left|\partial B^{1}\right|}{\left(\log \frac{R}{\rho}\right)^{n-1}} .
$$


By Hölder's and Sobolev's inequality with respect to the measure $p^{N} d x$ we have

$$
\begin{aligned}
\int_{B_{R}} & \left|\nabla\left(\phi u_{\varepsilon}\right)\right|^{2} p^{N-2} d x \\
\leq & (1+\beta) \int_{B_{R}}|\phi|^{2}\left|\nabla u_{\varepsilon}\right|^{2} p^{N-2} d x+\left(1+\frac{1}{\beta}\right) \int_{B_{R}}\left|u_{\varepsilon}\right|^{2}|\nabla \phi|^{2} p^{N-2} d x \\
\leq & (1+\beta) \int_{B_{R}}\left|\nabla u_{\varepsilon}\right|^{2} p^{N-2} d x \\
& +\left(1+\frac{1}{\beta}\right)\left(\int_{B_{R}}\left|u_{\varepsilon}\right|^{2^{*}} p^{N} d x\right)^{2 / 2^{*}}\left(\int_{B_{R}}|\nabla \phi|^{n}\right)^{2 / n} \\
\leq & \int_{B_{R}}\left|\nabla u_{\varepsilon}\right|^{2} p^{N-2} d x \\
& +\left[\beta+c\left(1+\frac{1}{\beta}\right)\left(\log \frac{R}{\rho}\right)^{-2(n-1) / n}\right] \int_{D}\left|\nabla u_{\varepsilon}\right|^{2} p^{N-2} d x
\end{aligned}
$$

for arbitrary $\beta>0$. Choosing $\beta$ small and $\rho$ small enough makes the second term smaller than a given $\delta>0$. Thus

$$
\begin{aligned}
\mu\left(\overline{B_{R}}\right)+\delta & =\limsup _{\varepsilon \rightarrow 0} \int_{B_{R}}\left|\nabla u_{\varepsilon}\right|^{2} p^{N-2} d x+\delta \\
& \geq \limsup _{\varepsilon \rightarrow 0} \int_{B_{R}}\left|\nabla\left(\phi u_{\varepsilon}\right)\right|^{2} p^{N-2} d x \\
& \geq\left(S+o\left(R^{0}\right)\right) \limsup _{\varepsilon \rightarrow 0}\left(\int_{B_{R}}\left|\phi u_{\varepsilon}\right|^{2^{*}} p^{N} d x\right)^{2 / 2^{*}} \\
& \geq\left(S+o\left(R^{0}\right)\right) \limsup _{\varepsilon \rightarrow 0}\left(\int_{B_{\rho}}\left|u_{\varepsilon}\right|^{2^{*}} p^{N} d x\right)^{2 / 2^{*}} \\
& \geq\left(S+o\left(R^{0}\right)\right) \nu_{j}^{2 / 2^{*}} .
\end{aligned}
$$

As $\delta \rightarrow 0$ and $R \rightarrow 0$ we get (32). Next we show that $\widetilde{\nu}=0$. Consider $\widetilde{u}_{\varepsilon}:=u_{\varepsilon}-u_{0}$. For a subsequence we have $\widetilde{u}_{\varepsilon} \rightarrow 0$ in $H_{0}^{1}\left(D, p^{N} d x\right)$ and

$$
\begin{aligned}
\left|\nabla \widetilde{u}_{\varepsilon}\right|^{2} p^{N-2} d x & \rightarrow \widetilde{\mu}_{0} \text { in } \mathcal{M}(\bar{D}), \\
\left|\widetilde{u}_{\varepsilon}\right|^{2^{*}} p^{N} d x & \rightarrow \widetilde{\nu}_{0} \text { in } \mathcal{M}(\bar{D}) .
\end{aligned}
$$

Choose an arbitrary closed set $\widetilde{D} \subset \bar{D}$ and a test-function $\phi \in C_{c}^{\infty}\left(\mathbb{R}^{N}\right)$ with $\phi=1$ on $\widetilde{D}$. By definition of $S_{p}(D)$ we have

$$
\begin{aligned}
S_{p}(D) & \leq \liminf _{\varepsilon \rightarrow 0} \frac{\int_{D}\left|\nabla\left(\phi \widetilde{u}_{\varepsilon}\right)\right|^{2} p^{N-2} d x}{\left(\int_{D}\left|\phi \widetilde{u}_{\varepsilon}\right|^{2^{*}} p^{N} d x\right)^{2 / 2^{*}}} \\
& \leq \frac{\int_{\bar{D}}|\phi|^{2} d \widetilde{\mu}_{0}}{\left(\int_{\bar{D}}|\phi|^{2^{*}} d \widetilde{\nu}_{0}\right)^{2 / 2^{*}}} \leq \frac{\int_{\bar{D}}|\phi|^{2} d \widetilde{\mu}_{0}}{\left(\widetilde{\nu}_{0}(\widetilde{D})\right)^{2 / 2^{*}}}
\end{aligned}
$$

since $\widetilde{u}_{\varepsilon} \rightarrow 0$ in $H_{0}^{1}\left(D, p^{N} d x\right)$. Taking the infimum over all such $\phi$ yields

$$
S_{p}(D)\left(\widetilde{\nu}_{0}(\widetilde{D})\right)^{2 / 2^{*}} \leq \widetilde{\mu}_{0}(\widetilde{D})
$$


In particular the measure $\widetilde{\nu}_{0}$ is absolutely continuous with respect to $\widetilde{\mu}_{0}$. By the Radon-Nikodým theorem

$$
\widetilde{\nu}_{0}=\frac{d \widetilde{\nu}_{0}}{d \widetilde{\mu}_{0}} \widetilde{\mu}_{0}
$$

with $\frac{d \widetilde{\nu}_{0}}{d \widetilde{\mu}_{0}} \in L^{1}\left(\bar{D}, \widetilde{\mu}_{0}\right)$. By Lebesgue's differentiation theorem,

$$
\frac{d \widetilde{\nu}_{0}}{d \widetilde{\mu}_{0}}(x)=\lim _{\rho \rightarrow 0} \frac{\widetilde{\nu}_{0}\left(\overline{B_{x}^{\rho}}\right)}{\widetilde{\mu}_{0}\left(\overline{B_{x}^{\rho}}\right)}
$$

for $\widetilde{\mu}_{0}$ almost every $x \in \bar{D}$. With $\widetilde{D}=\overline{B_{x}^{\rho}}$ inequality (33) yields

$$
\begin{aligned}
S_{p}(D)\left(\frac{d \widetilde{\nu}_{0}}{d \widetilde{\mu}_{0}}(x)\right)^{2 / 2^{*}} & =\lim _{\rho \rightarrow 0} \frac{S_{p}(D)\left(\widetilde{\nu}_{0}\left(\overline{B_{x}^{\rho}}\right)\right)^{2 / 2^{*}}}{\left(\widetilde{\mu}_{0}\left(\overline{B_{x}^{\rho}}\right)\right)^{2 / 2^{*}}} \\
& \leq \lim _{\rho \rightarrow 0}\left(\widetilde{\mu}_{0}\left(\overline{B_{x}^{\rho}}\right)\right)^{1-2 / 2^{*}}=0
\end{aligned}
$$

except if $x$ is an atom of $\widetilde{\mu}_{0}$. In the latter case $\frac{d \widetilde{\nu}_{0}}{d \widetilde{\mu}_{0}}(x)=0$ except if $x$ is also an atom of $\widetilde{\nu}_{0}$. By the Brézis-Lieb lemma the atoms of $\widetilde{\nu}_{0}$ and $\widetilde{\nu}$ are the same, namely $\left\{x_{1} \ldots x_{J}\right\}$. We conclude that $\frac{d \widetilde{\nu}_{0}}{d \widetilde{\mu}_{0}}=0$ as a function in $L^{1}\left(\bar{D} \backslash\left\{x_{1} \ldots x_{J}\right\}, d \widetilde{\mu}_{0}\right)$ and therefore $\widetilde{\nu}_{0}=0$ in $D \backslash\left\{x_{1} \ldots x_{J}\right\}$, i.e. $\widetilde{\nu}=0$ as desired. As to the alternative, define

$$
\mu_{0}:=\int_{D}\left|\nabla u_{0}\right|^{2} p^{N-2} d x, \quad \nu_{0}:=\int_{D}\left|u_{0}\right|^{2^{*}} p^{N} d x .
$$

Then $\mu(\bar{D})=S_{p}(D)$, and Proposition 16 implies

$$
\begin{gathered}
\left(\sum_{j=0}^{J} \frac{\mu_{j}}{S_{p}(D)}\right)^{2^{*} / 2} \leq\left(\frac{\mu(\bar{D})}{S_{p}(D)}\right)^{2^{*} / 2}=1=\sum_{j=0}^{J} \nu_{j} \\
\leq\left(\frac{\mu_{0}}{S_{p}(D)}\right)^{2^{*} / 2}+\sum_{j=1}^{J}\left(\frac{\mu_{j}}{S}\right)^{2^{*} / 2} \leq \sum_{j=0}^{J}\left(\frac{\mu_{j}}{S_{p}(D)}\right)^{2^{*} / 2}
\end{gathered}
$$

by (32) and $\widetilde{\mu} \geq 0$. By strict convexity of the function $\tau \mapsto \tau^{2^{*} / 2}$ on $(0,1]$ only one of the $\mu_{j}$ 's can be nonzero. Therefore compactness or concentration must hold.

From this alternative we derive the following compactness criterion.

Theorem 18 (Compactness criterion). 1. If $S_{p}(D)<S$, then $S_{p}(D)$ is attained.

2. If $0<S_{p}(D)=S$, then either $S_{p}(D)$ is attained or every minimizing sequence for $S_{p}(D)$ has a subsequence which concentrates at some point in $\bar{D}$. Moreover for every point in $\bar{D}$ there is a maximizing sequence concentrating at this point.

Proof. We apply the concentration-compactness alternative (Theorem 17) to a minimizing sequence $\left(u_{\varepsilon}\right)$ in $E$ for $S_{p}(D)$. For a subsequence of $\left(u_{\varepsilon}\right)$ the weak limits $u_{0}$, $\mu$ and $\nu$ exist. If $S_{p}(D)<S$, concentration cannot occur because the last inequality in (34) would be strict, which is impossible unless $\mu_{j}=0$ for $j \geq 1$.

The first assertion was also obtained by Egnell [10]. 
7.2. Dirichlet integral. We relate the Dirichlet integral for the metric $p|d x|$ with the Euclidean one. Our formula involves the scalar curvature $K$ of $p|d x|$, which satisfies

$$
\begin{aligned}
-\Delta p^{\frac{N-2}{2}} & =\frac{N-2}{4(N-1)} K p^{\frac{N+2}{2}} \quad(N \geq 3), \\
-\frac{\nabla}{p} \cdot \frac{\nabla p}{p} & =K p \quad(N=2) .
\end{aligned}
$$

Note that the product $K p^{2}$ is dimensionless, i.e. invariant under dilations. The scalar curvature of the spherical and hyperbolic metric is

$$
K_{ \pm}= \pm N(N-1) .
$$

Lemma 19. For $p \in C^{2}(D)$ and $v \in E$ put

$$
v_{0}:=v p^{\frac{N-2}{2}},
$$

which is in

$$
E_{0}:=\left\{v_{0} \in H_{0}^{1}(D, d x): \int_{D}\left|v_{0}\right|^{2^{*}} d x=1\right\}
$$

Then

$$
\int_{D}|\nabla v|^{2} p^{N-2} d x=\int_{D}\left|\nabla v_{0}\right|^{2} d x-\frac{N-2}{4(N-1)} \int_{D} v_{0}^{2} K p^{2} d x .
$$

Proof. The conformal gradient of $v$ is

$$
\frac{\nabla v}{p}=\frac{\nabla v_{0}}{p^{\frac{N}{2}}}-\frac{N-2}{2} v_{0} \frac{\nabla p}{p^{\frac{N+2}{2}}} .
$$

Therefore

$$
|\nabla v|^{2} p^{N-2}=\left|\nabla v_{0}\right|^{2}-\frac{N-2}{2} \nabla v_{0}^{2} \cdot \frac{\nabla p}{p}+\left(\frac{N-2}{2}\right)^{2} v_{0}^{2}\left|\frac{\nabla p}{p}\right|^{2} .
$$

Integration of the mixed term gives

$$
\frac{N-2}{2} \int_{D} v_{0}^{2} \nabla \cdot \frac{\nabla p}{p} d x
$$

where

$$
\frac{N-2}{2} \nabla \cdot \frac{\nabla p}{p}=\nabla \cdot \frac{\nabla p^{\frac{N-2}{2}}}{p^{\frac{N-2}{2}}}=-\frac{N-2}{4(N-1)} K p^{2}-\left(\frac{N-2}{2}\right)^{2}\left|\frac{\nabla p}{p}\right|^{2},
$$

as follows from (35). This yields the assertion.

Based on a Pohozaev identity, we prove the following nonexistence result.

Theorem 20. If $D$ is starshaped with respect to the origin and

$$
2 K p^{2}+\nabla\left(K p^{2}\right) \cdot x \leq 0 \text { in } D,
$$

then (31) has no solution except the trivial one.

Proof. If $v$ is a solution of (31) then $v_{0}$ solves

$$
\begin{aligned}
\Delta v_{0}+\frac{N-2}{4(N-1)} K p^{2} v_{0}+S_{p}(D) v_{0}^{2^{*}-1} & =0 \text { in } D, \\
v_{0} & =0 \text { on } \partial D,
\end{aligned}
$$


as follows from Lemma 19 . Testing this equation with $\nabla v_{0} \cdot x$ and replacing $\int_{D}\left|\nabla v_{0}\right|^{2}-\int_{D}\left|v_{0}\right|^{2^{*}}$ by $\frac{N-2}{4(N-1)} \int_{D} K p^{2} v_{0}^{2}$ in the resulting Pohozaev identity yields

$$
\frac{N-2}{4(N-1)} \int_{D}\left(2 K p^{2}+\nabla\left(K p^{2}\right) \cdot x\right) v_{0}^{2} d x=\int_{\partial D}\left|\nabla v_{0}\right|^{2} x \cdot \nu
$$

where $\nu$ denotes the exterior unit normal of $D$. By assumption the left hand side is negative, the right hand side positive. Thus $v_{0}=0$.

The only difference between the above and the standard Pohozaev identity (see e.g. Struwe [22]) is the second term on the left, due to the $x$-dependence of the linear term. A more general Pohozaev identity with $x$-dependent coefficients can be found in Egnell [10, Lemma 4].

7.3. Best Sobolev constant of hyperbolic domains. The general nonexistence result applies to spherical balls.

Corollary 21. For a ball $B_{R}$ in $H^{N}$ with $N \geq 3$ problem (31) has no positive solution except the trivial one.

Proof. This follows from Theorem 20 because

$$
2 K_{-} p_{-}^{2}+\nabla\left(K_{-} p_{-}^{2}\right) \cdot x=-2 p_{-}^{2}\left(1+p_{-}|x|^{2}\right)<0 .
$$

Theorem 22. Let $D$ be a hyperbolic domain. Then $S_{-}(D)=S$ and $S_{-}(D)$ is not achieved. Every point in $\bar{D}$ can be a concentration point of a minimizing sequence for $S_{-}(D)$.

Proof. Suppose that $S_{-}(D)$ is achieved by some minimizer $u$. By Proposition 16, hyperbolic symmetrization with respect to the measure $p_{-}^{N} d x$ (Baernstein [4]), and the definition of $S_{-}(D)$ we get

$$
S \geq S_{-}(D) \geq \int_{D^{*}}\left|\nabla u^{*}\right|^{2} p_{-}^{N-2} d x \geq S_{-}\left(D^{*}\right) .
$$

If $S_{-}\left(D^{*}\right)<S$ then $S_{-}\left(D^{*}\right)$ would be attained by the compactness criterion (Theorem 18). A minimizer would be a nontrivial radial solution of (31), which is impossible by Corollary 21. Therefore $S_{-}\left(D^{*}\right) \geq S$. Thus the above inequalities imply that $u^{*}$ is a minimizer for $S_{-}\left(D^{*}\right)$, which is again impossible by Corollary 21 . Hence $S_{-}(D)$ is not attained.

7.4. Best Sobolev constant of spherical domains. The situation is different for spherical domains.

Theorem 23. The best Sobolev constant $S_{+}\left(B_{R}\right)$ of a ball in $S^{N}$ satisfies:

1. $S_{+}\left(B_{R}\right)$ is a nonincreasing function of $R$.

2. If $N \geq 4$ then

$$
S_{+}\left(B_{R}\right)<S
$$

for every $R>0$.

3. In three dimensions there is a critical radius $R_{c}>0$ such that

$$
\begin{aligned}
& S_{+}\left(B_{R}\right)<S \text { for } R>R_{c}, \\
& S_{+}\left(B_{R}\right)=S \text { for } R<R_{c} .
\end{aligned}
$$

4. $S_{+}\left(B_{R}\right) \rightarrow S$ as $R \rightarrow 0$. 
5. $S_{+}\left(S^{N}\right)=0$.

Proof. $\quad$ 1. If the domain $D$ increases, then the admissible set $E$ becomes larger and the infimum (30) decreases.

2. By (29) and Lemma 19 we have

$$
\begin{aligned}
0 & <\frac{S}{\left(1+R^{2}\right)^{N-2}} \leq S_{+}\left(B_{R}\right) \\
& \leq \inf _{v_{0} \in E_{0}}\left(\int_{B_{R}}\left|\nabla v_{0}\right|^{2} d x-\frac{N(N-2)}{\left(1+R^{2}\right)^{2}} \int_{B_{R}} v_{0}^{2} d x\right)=: \quad \widetilde{S} .
\end{aligned}
$$

The assertion follows from a result of Brézis and Nirenberg [9, Lemma 1.1]. It says that for $N \geq 4$ and every positive constant in front of the second integral one has $\widetilde{S}<S$. An extension of the Brézis-Nirenberg result is also given by Egnell [10].

3. We take the capacity potentials

$$
v_{0}(x):= \begin{cases}c_{R} & (0 \leq|x| \leq R-1), \\ c_{R}\left(\frac{1}{|x|-1}-\frac{1}{R-1}\right) & (R-1 \leq|x| \leq R)\end{cases}
$$

as test functions in $E_{0}$. A rough estimate gives

$$
S_{+}\left(B_{R}\right) \leq \frac{c}{R}<S
$$

for $R$ large enough. On the other hand

$$
S_{+}\left(B_{R}\right) \geq \inf _{v_{0} \in E_{0}}\left(\int_{B_{R}}\left|\nabla v_{0}\right|^{2} d x-3 \int_{B_{R}} v_{0}^{2} d x\right)
$$

by Lemma 19. The result of Brézis and Nirenberg for the three-dimensional case [9, Theorem 1.2] implies that the right hand side equals $S$ if $3 \leq\left(\frac{\pi}{2 R}\right)^{2}$.

4. By Hölder's inequality functions in $E_{0}$ satisfy

$$
\int_{B_{R}} v_{0}^{2} p_{+}^{2} d x \leq\left(\int_{B_{R}} p_{+}^{N} d x\right)^{2 / N} .
$$

By Lemma 19

$$
\begin{aligned}
S_{+}\left(B_{R}\right) & =\inf _{v_{0} \in E_{0}}\left(\int_{B_{R}}\left|\nabla v_{0}\right|^{2} d x-\frac{N(N-2)}{4} \int_{B_{R}} v_{0}^{2} p_{+}^{2} d x\right) \\
& \geq S-\frac{N(N-2)}{4}\left(\int_{B_{R}} p_{+}^{N} d x\right)^{2 / N},
\end{aligned}
$$

which proves the assertion.

5. We use the test function

$$
v_{k}(x):=\frac{c_{k}}{\left(k^{2}+|x|^{2}\right)^{\frac{N-2}{2}}}
$$

and let $k \rightarrow \infty$.

Next we try to determine the critical radius $R_{c}$ as it appears in the threedimensional spherical case numerically. An extremal function $v$ for $S_{ \pm}\left(B_{R}\right)$ corresponds to a radial solution $v_{0}$ of $(37)$. The unknown coefficient $S_{ \pm}\left(B_{R}\right)$ can be 


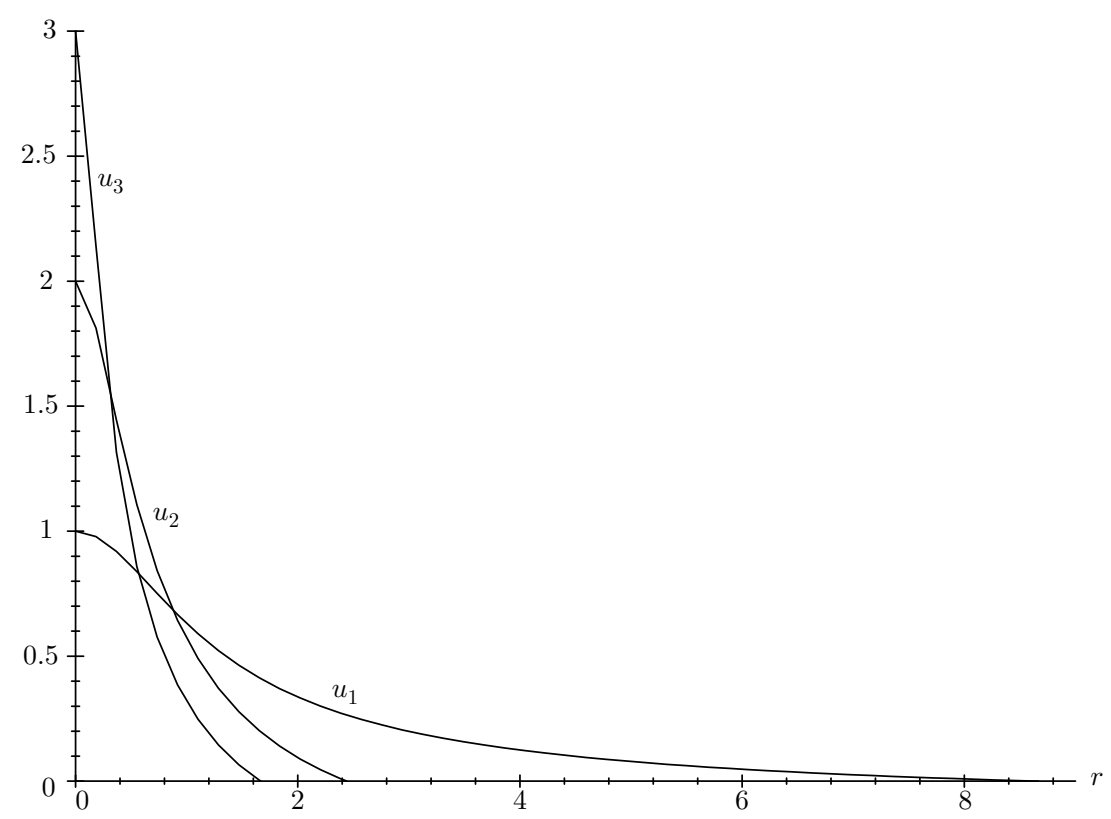

Figure 1. Solutions of (38) for $N=3$, spherical metric, $u(0)=1,2,3$

eliminated by scaling $u(|x|):=\alpha v_{0}(x)$ so that (37) becomes

$$
\begin{aligned}
u^{\prime \prime}+\frac{N-1}{r} u^{\prime} \pm N(N-2) \frac{u}{\left(1 \pm r^{2}\right)^{2}}+u^{2^{*}-1} & =0 \text { on }(0, R), \\
u^{\prime}(0) & =0, \\
u(R) & =0 .
\end{aligned}
$$

The factor $\alpha$ and the best Sobolev constant are determined by the normalization of $v_{0}$ :

$$
\begin{aligned}
\alpha^{2^{*}} & =|\partial B| \int_{0}^{R} r^{N-1}|u(r)|^{2^{*}}, \\
S_{+}\left(B_{R}\right) & =\alpha^{2^{*}-2} .
\end{aligned}
$$

Instead of $R$ we fix $u(0)$ so that we can use a standard ODE solver to approximate the corresponding solution $u_{u(0)}$. The radius $R$ is then determined as the first positive zero of $u_{u(0)}$. Figure 1 shows several solutions in three dimensions.

As $u(0) \nearrow \infty$ we find $R \searrow R_{c}$ as expected (Figure 2). Concentration implies $u_{k} \rightarrow 0$ in $H_{0}^{1}\left(D, p^{N} d x\right)$ as $k \rightarrow \infty$. In the radial case $u_{k} \rightarrow 0$ locally uniformly away from the origin. The experiment suggests $R_{c}=1$, which is the radius of the half sphere. Figures 3 and 4 show that this phenomenon does not occur in higher dimensions and for the hyperbolic metric. In the spherical case of dimension $N \geq 4$ we have $R \searrow 0$ as $u(0) \nearrow \infty$. In the hyperbolic case no solution has a positive zero. Finally, we state our main result for spherical domains.

Theorem 24. Let $D$ be a spherical domain.

1. If $N \geq 4$, then $S_{+}(D)$ is attained.

2. If $N=3$ and $\max r>R_{c}$ with $R_{c}$ as in Theorem 23, then $S_{+}(D)$ is attained. 


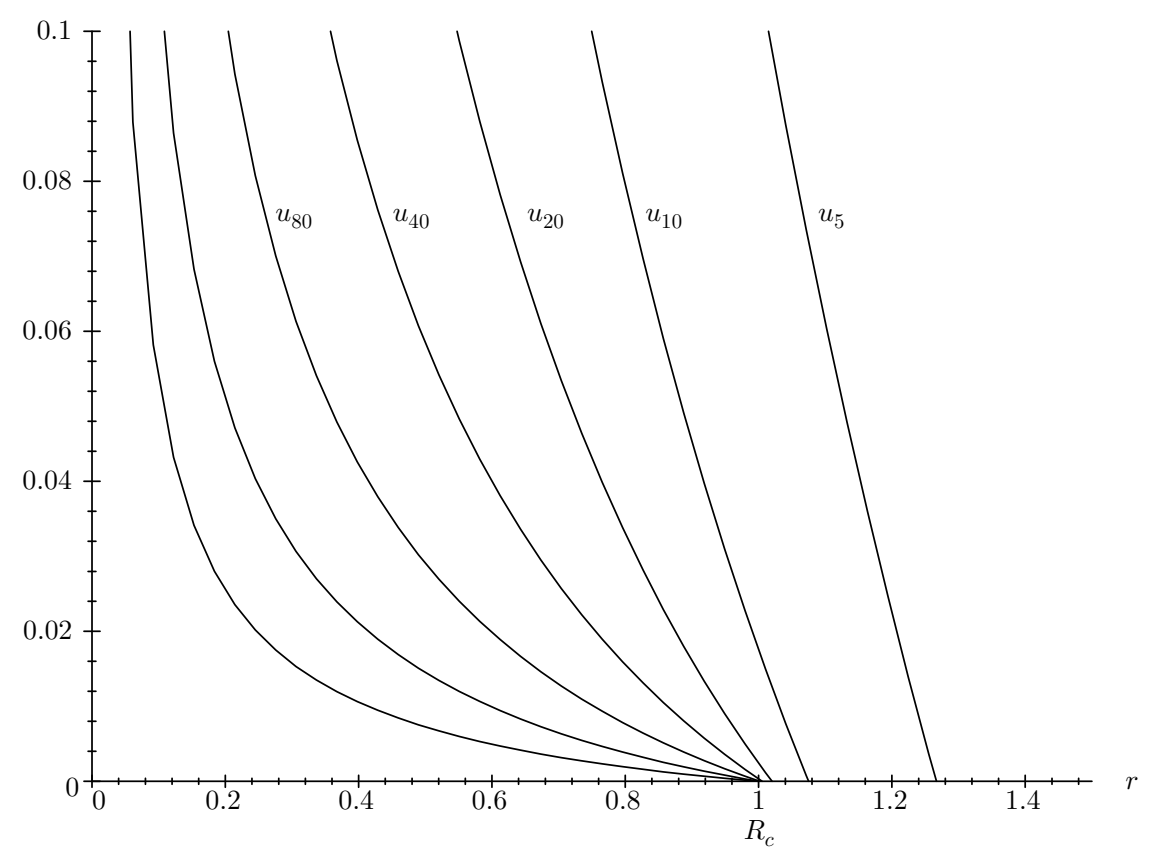

Figure 2. Solutions of (38) for $N=3$, spherical metric in the vicinity of $R_{c}, u(0)=5,10,20,40,80,160,320$

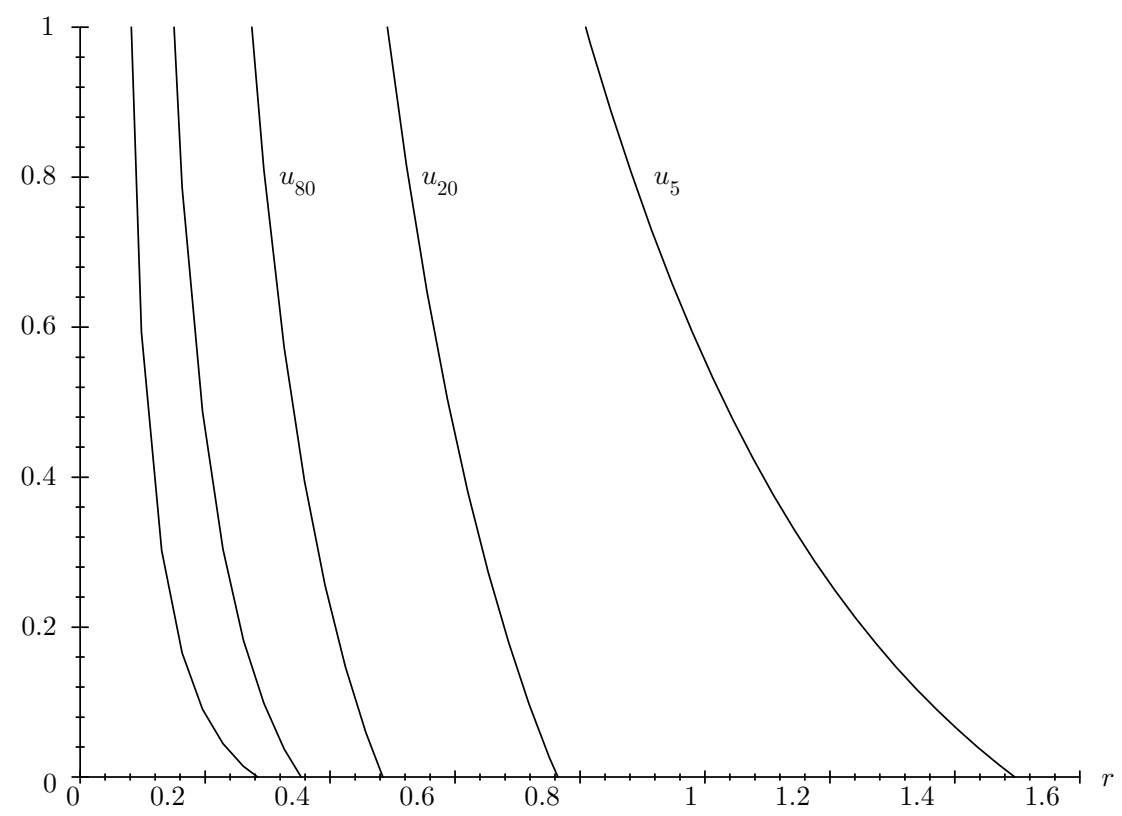

Figure 3. Solutions of (38) for $N=4$, spherical metric, $u(0)=$ $5,20,80,320,1280$ 


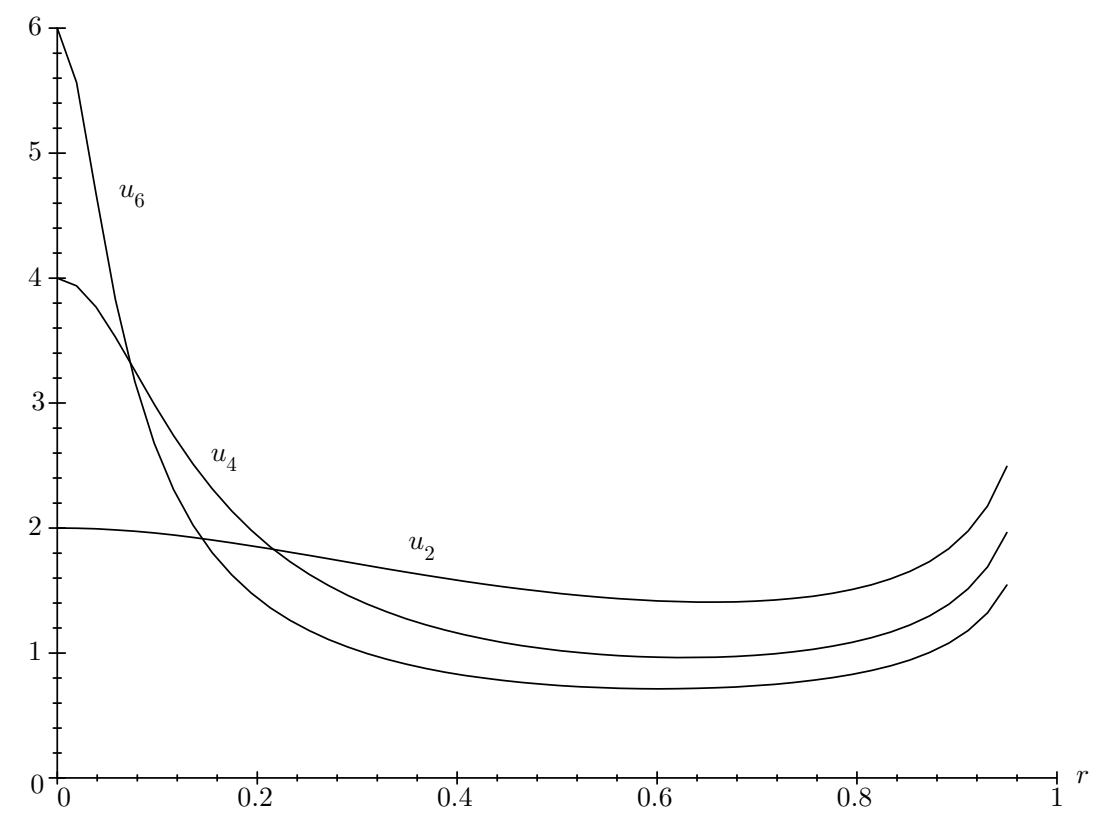

Figure 4. Solutions of (38) for $N=3$, hyperbolic metric, $u(0)=2,4,6$

3. If $N=3$ and $m(D)<m\left(B_{R_{c}}\right)$ with $m$ as in $(16)$, then $S_{+}(D)=S$.

Proof. 1. Consider a radial minimizer $u$ in the ball $B_{\max r}$ and let $U$ be its harmonic transplantation to $D$ centered at a harmonic center. By Theorem 14 and Theorem 23 we have

$$
\begin{aligned}
S_{+}(D) & \leq \frac{\int_{D}|\nabla U|^{2} p^{N-2} d x}{\left(\int_{D}|U|^{2^{*}} p^{N} d x\right)^{2 / 2^{*}}} \\
& \leq \frac{\int_{B_{\max r}}|\nabla u|^{2} p^{N-2} d x}{\left(\int_{B_{\max r}}|u|^{2^{*}} p^{N} d x\right)^{2 / 2^{*}}}=S_{+}\left(B_{\max r}\right)<S .
\end{aligned}
$$

The assertion follows from the compactness criterion (Theorem 18).

2. The above inequality and assertion 3 of Theorem 23 yields the claim.

3. This follows by symmetrization $S_{+}(D) \geq S_{+}\left(D^{*}\right)$ and 3 of Theorem 23 .

The discussion of the variational problem (29) can be extended to more general conformal metrics $p|d x|$. By Lemma 19

$$
\begin{aligned}
& \inf _{v_{0} \in E_{0}}\left(\int_{D}\left|\nabla v_{0}\right|^{2} d x-\gamma_{1} \int_{D} v_{0}^{2} d x\right) \\
& \quad \leq S_{p}(D) \leq \inf _{v_{0} \in E_{0}}\left(\int_{D}\left|\nabla v_{0}\right|^{2} d x-\gamma_{0} \int_{D} v_{0}^{2} d x\right)
\end{aligned}
$$

where

$$
\gamma_{0(1)}=\min _{D}\left(\max _{D}\right) \frac{N-2}{4(N-1)} p^{2} K
$$


with $K$ as in (35). Applying the results of Brézis and Nirenberg [9] and Proposition 16 , we see that there is a critical value $\gamma_{c} \geq 0$ such that $S_{p}(D)<S$ if $\gamma_{0}>\gamma_{c}$ and $S_{p}(D)=S$ if $\gamma_{1}<\gamma_{c}$. If $S_{p}(D)=S$, then according to Theorem 18 two cases can occur. Either there is a minimizer, or every minimizing sequence concentrates.

A different aspect of critical Sobolev inequalities has been investigated by Hebey and Vaugon [13]. On a general Riemannian manifold $(M, g)$ one has

$$
\left(\int_{M}|u|^{2^{*}} d v(g)\right)^{2 / 2^{*}} \leq\left(\frac{1}{S}+\delta\right) \int_{M}\left|\nabla_{g} u\right|^{2} d v(g)+C_{\delta} \int_{M} u^{2} d v(g)
$$

for every $\delta>0$. Hebey and Vaugon show that this inequality also holds for $\delta=0$. This is what they call attainment (following Aubin), whereas we use attainment for existence of an extremal function. From our results we derive the following additional information on the best limiting constant $C_{0}$.

Corollary 25. The constant $C_{0}$ in (39) with $\delta=0$ satisfies:

1. If $S_{p}(D)=S$ then $C_{0}=0$.

2. Otherwise $S_{p}(D)<S$ and

$$
C_{0} \geq \lambda_{1}\left(\frac{1}{S_{p}(D)}-\frac{1}{S}\right),
$$

where $\lambda_{1}>0$ denotes the principal Dirichlet eigenvalue of the corresponding elliptic operator.

Proof. 1. This holds by definition of $S_{p}(D)$.

2. If $S_{p}(D) \neq S$ then $S_{p}(D)<S$ by Proposition 16 and Theorem 18. An extremal function $u \neq 0$ satisfying

$$
\left(\int_{D}|u|^{2^{*}} p^{N} d x\right)^{2 / 2^{*}}=\frac{1}{S_{p}(D)} \int_{D}|\nabla u|^{2} p^{N-2} d x
$$

exists. Taking the difference with the Hebey-Vaugon inequality

$$
\left(\int_{D}|u|^{2^{*}} p^{N} d x\right)^{2 / 2^{*}} \leq \frac{1}{S} \int_{D}|\nabla u|^{2} p^{N-2} d x+C_{0} \int_{D} u^{2} p^{N} d x
$$

we obtain

$$
\begin{aligned}
0 & \geq\left(\frac{1}{S_{p}(D)}-\frac{1}{S}\right) \int_{D}|\nabla u|^{2} p^{N-2} d x-C_{0} \int_{D} u^{2} p^{N} d x \\
& \geq\left(\frac{1}{S_{p}(D)}-\frac{1}{S}-\frac{C_{0}}{\lambda_{1}}\right) \int_{D}|\nabla u|^{2} p^{N-2} d x .
\end{aligned}
$$

The factor in parentheses has to be negative.

Open problems. If $S_{p}(D)=S$, we do not know whether the best Sobolev constant is attained, or whether minimizing sequences concentrate, or both. The radial case in $\mathbb{R}^{3}$ has been analyzed by Atkinson and Peletier [2]. They found that the Euler equation of

$$
\inf \left\{\frac{\int_{B}|\nabla u|^{2} d x-\frac{\pi^{2}}{4} \int_{B} u^{2} d x}{\left(\int_{B} u^{6}\right)^{1 / 3}}: u \in H_{0}^{1}(B)\right\}
$$

has no radial solution. By the concentration compactness alternative, every minimizing sequence concentrates. 


\section{REFERENCES}

[1] Ahlfors L. V., Möbius transformations in several dimensions, Ordway Lecture in Mathematics, University of Minnesota, 1981. MR 84m:30028

[2] Atkinson F. V., Peletier L. A., Large solutions of elliptic equations involving critical exponents, Asymptotic Anal. 1 (1988), 139-160. MR 89i:35004

[3] Aubin T., Prescribed scalar curvature and the method of isometry-concentration, Symposia Math. 35 (1994), 37-45. MR 95h:53051

[4] Baernstein II A., A uniform approach to symmetrization, Symposia Math. 35 (1994), 47-91. MR 96e:26019

[5] Bandle C., Isoperimetric inequalities and applications, Pitman Monographs and Studies in Mathematics (1980). MR 81e:35095

[6] Bandle C., Flucher M., Harmonic radius and concentration of energy; hyperbolic radius and Liouville's equations $\Delta U=e^{U}$ and $\Delta U=U^{\frac{n+2}{n-2}}$, SIAM Review 38-2 (1996), 191-238. MR 97b:35046

[7] Bernstein A., Über die isoperimetrische Eigenschaft des Kreises auf der Kugeloberfläche und in der Ebene, Math. Ann. 60 (1905), 117-136.

[8] Brézis H., Lieb E., A relation between pointwise convergence of functions and convergence of functionals, Proc. Amer. Math. Soc. 88.3 (1983), 486-490. MR 84e:28003

[9] Brézis H., Nirenberg L., Positive solutions of nonlinear elliptic equations involving critical Sobolev exponents, Comm. Pure Appl. Math. 36 (1983), 437-477. MR 84h:35059

[10] Egnell H., Semilinear elliptic equations involving critical Sobolev exponents, Arch. Rational Mech. Anal. 104 (1988), 27-56. MR 90e:35068

[11] Flucher M., Müller S., Concentration of low energy extremals, Ann. Inst. H. Poincaré Analyse Non Linéaire, to appear.

[12] Gasser T., Hersch J., Über die Eigenwerte einer mehrfach zusammenhängenden Membran: Erweiterung von isoperimetrischen Sätzen von Pólya und Szegö, ZAMP 19 (1968), 672-675. MR 38:2672

[13] Hebey E., Vaugon M., Meilleures constantes dans le théorème d'inclusion de Sobolev, Ann. Inst. H. Poincaré - Analyse Non Linéaire 13.1 (1996), 57-93. MR 96m:46054

[14] Hersch J., Transplantation harmonique, transplantation par modules et théorèmes isopérimétriques, Comment. Math. Helvetici 44.3 (1969), 354-366. MR 40:837

[15] Lions P.L., The concentration-compactness principle in the calculus of variations, The limit case, Part 1, Rev. Mat. Iberoamericana 1.1 (1985), 145-201. MR 87c:49007

[16] Miranda C., Partial Differential Equations of Elliptic Type, Springer 1970. MR 44:1924

[17] Payne L. E., Some isoperimetric inequalities in the torsion problem for multiply connected regions, Studies in Mathematical Analysis and Related Topics: Essays in Honor of G. Pólya, Stanford University Press (1962), 270-280. MR 29:774

[18] Pólya G., Schiffer M., Convexity of functionals by transplantation, J. Analyse Mathém. 3 (1954). MR 16:591e

[19] Pólya G., Szegő G., Isoperimetric inequalities in mathematical physics, Princeton University Press (1951). MR 13:270d

[20] Schmidt E., Die Brunn-Minkowskische Ungleichung und ihr Spiegelbild sowie die isoperimetrische Eigenschaft der Kugel in der euklidischen und nicht-euklidischen Geometrie I, II, Math. Nachr. 1 (1948), 81-157; 2 (1949), 171-244. MR 10:471d; MR 11:534l

[21] Sperner E., Zur Symmetrisierung von Funktionen auf Sphären, Math. Z. 134 (1973), 317-327. MR 49:5310

[22] Struwe M., Variational methods, Springer-Verlag (1990), 2nd ed., 1996. MR 92b:49002

[23] Weinberger H., Symmetrization in uniformly elliptic problems, Studies in Mathematical Analysis and Related Topics: Essays in Honor of G. Pólya, Stanford University Press (1962), 424-428. MR 26:2726

Universität Basel, Mathematisches Institut, Rheinsprung 21, CH-4051 Basel, Schweiz E-mail address: bandle@math.unibas.ch

Université de Haute Alsace, Faculté des Sciences Techniques, 4 rue des Frères Lumière, F-68093 Mulhouse Cédex, France

E-mail address: A.Brillard@univ--mulhouse.fr

Universität Basel, Mathematisches Institut, Rheinsprung 21, CH-4051 Basel, Schweiz

E-mail address: flucher@math.unibas.ch 